\title{
Panarteritis nodosa cutánea, presentación de un caso con lesiones en localización atípica
}

\author{
Ramiro Puerta Franchi ${ }^{1}$, Mariel Olguin ${ }^{2}$, Roberto Gavazzi ${ }^{3}$ \\ ${ }^{1}$ Médico Reumatólogo del Sanatorio de La Trinidad San Isidro. ${ }^{2}$ Médica Clínica del Sanatorio de La Trinidad San Isidro. ${ }^{3}$ Jefe de \\ Internación del Sanatorio de La Trinidad San Isidro. Buenos Aires.
}

\begin{abstract}
Resumen
La Panarteritis Nodosa Cutánea es una rara entidad caracterizada por nódulos subcutáneos, de curso crónico, benigno y recurrente. La mayoría de los pacientes presentan lesiones en miembros inferiores, y un tercio en los miembros superiores. Además existen manifestaciones extracutáneas como miositis 0 artritis.

Presentamos el caso de una paciente que fue internada por tumefacción, debilidad muscular y dolor incapacitante en ambos hombros y nódulos subcutáneos en hombros y cara, a quien le habían diagnosticado panarteritis nodosa en la infancia, pero que siempre presentó lesiones en localizaciones atípicas, respetando los miembros inferiores.
\end{abstract}

\section{Abstract}

Cutaneous Polyarteritis Nodosa is a rare entity characterized by subcutaneous nodules, as well as chronic, benign and recurrent course. Most patients have lesions on the lower limbs, and one third on the upper limbs. In addition there are extracutaneous manifestations such as myositis or arthritis.

We present a case of a patient who was hospitalized for swelling, muscle weakness and disabling pain in both shoulders and subcutaneous nodules in the shoulders and face, who had been diagnosed with polyarteritis nodosa in infancy and always had lesions in atypical locations, respecting the lower limbs.

\section{Introducción}

La Panarteritis Nodosa Cutánea fue descripta en 1931 por Lindberg. Es una entidad caracterizada por nódulos subcutáneos, de curso crónico, benigno y recurrente ${ }^{1}$. Presentamos el caso de una paciente que cursó internación en nuestra institución por un cuadro compatible y con lesiones típicas, aunque de rara localización.

\section{Caso clínico}

Paciente de 18 años, mujer, oriunda de la provincia de Buenos Aires, Argentina, ingresa por tumefacción, debilidad muscular y dolor incapacitante en ambos hombros a predominio del derecho y nódulos subcutáneos en hombros y cara (Figura 1).

A los 9 años presentó nódulos subcutáneos en miembros superiores, de los cuales se tomó muestra y se diagnosticó panarteritis nodosa por biopsia compatible. Por esto recibió tratamiento con corticoides y metotrexato, los cuales suspendió meses previos a la consulta.

Durante la internación actual se indica tratamiento con AINE y opioides.

En los análisis de laboratorio presentaba eritrosedimentación, CPK y proteína C reactiva elevadas, sin alteraciones en la función renal ni hepática, sedimento urinario normal. FAN, Sm, RNP, Ro, La, FR, ANCA, PR3 y MPO negativos.

Se toman muestras de uno de los nódulos y de músculo deltoides.

Se interpreta el cuadro como miositis en contexto de panarteritis nodosa y se decide iniciar tratamiento con meprednisona $1 \mathrm{mg} / \mathrm{kg} /$ día, con buena respuesta.

Cuatro meses posteriores a la externación es ingresada por presentar nuevamente dolores, luego de haber descendido la dosis de esteroides a la mitad. En esta última internación se recibe resultado de biopsia de músculo

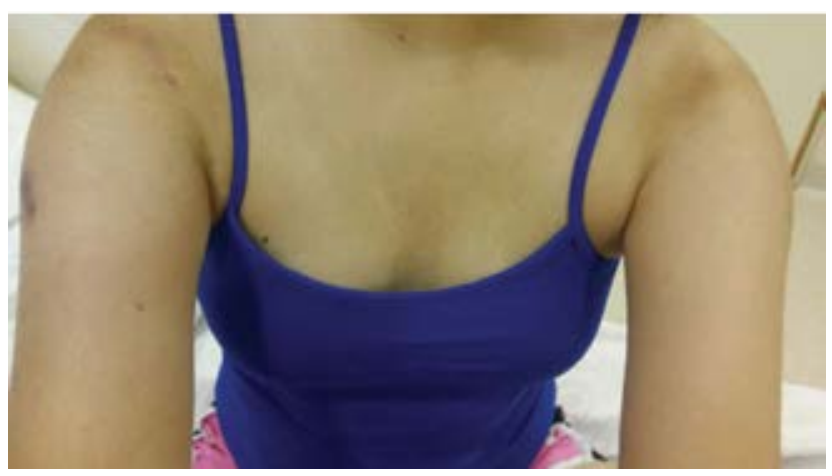

Figura 1. Obsérvese la asimetría de miembros, así como el tamaño aumentado de ambos brazos tomando como referencia el tórax. 
que informa infiltrado de mononucleares perimisial y perivascular con compromiso de la pared vascular. También se recibe resultado de biopsia de nódulo subcutáneo que informa granuloma inflamatorio no caseoso con inflamación crónica reagudizada. Compromiso de la pared vascular.

Se decide aumentar nuevamente la dosis de esteroides y agregar metotrexato al plan, interpretando el cuadro como una recaída de panarteritis nodosa cutánea.

\section{Discusión}

La Panarteritis Nodosa Cutánea fue descripta en 1931 por Lindberg. Es una entidad caracterizada por nódulos subcutáneos, de curso crónico, benigno y recurrente ${ }^{1}$. Verbou la describe en 1980 como una vasculitis que se limita a la piel, músculos y articulaciones, y se caracteriza por la ausencia de lesiones viscerales².

En una serie de 79 casos, los cuales fueron evaluados en promedio durante 6 a 9 años, describieron entre los pacientes que no habían desarrollado úlceras: edema de miembros inferiores en el $60 \%$, VSG elevada en el $60 \%$, Livedo Reticularis en el 50\%, nódulos subcutáneos en el $100 \%$. Además todos eran negativos para HCV y HBV. Durante el seguimiento ninguno presentó compromiso renal ${ }^{1}$.

En otra serie, de 16 pacientes, describieron lesiones nodulares y eritema indurado en el antebrazo en 2 casos, un caso en el brazo y otro en la mano ${ }^{3}$.

En una serie de 7 casos sugieren que aunque esta enfermedad se limita a la piel, puede presentar miositis local, con dolor circunscripto y rigidez ${ }^{4}$. En esta misma serie, el único caso descripto que suspendió el tratamiento con esteroides, presentó una recaída del cuadro.

En un trabajo realizado en Brasil, obtuvieron como resultados una relación hombre:mujer de 1:3,4, una edad media de 39,4 años (con un rango etario de 9 a 61 años), afección de los miembros inferiores en el 100\% de los casos y de los miembros superiores en el 27,3\%, la lesión más frecuente fue la úlcera $(63,7 \%)$ y los nódulos subcutáneos se hallaron en el $50 \%{ }^{5}$.

En una revisión refieren que el $97 \%$ de los pacientes presenta afección de miembros inferiores, $33 \%$ de miembros superiores y $8 \%$ de tronco. También comentan que el $31,2 \%$ de los pacientes presenta mialgias 6 . En este trabajo además hacen referencia a las recaídas en contexto del descenso de la dosis de esteroides.

El caso que presentamos cobra interés ya que si bien presenta las lesiones y el curso típico de la panarteritis nodosa cutánea, las mismas se presentan en una localización poco o nada frecuente, sin comprometer los miembros inferiores, los cuales se vieron afectados en todos los casos que encontramos en la literatura.

Cabe agregar que la definición de la enfermedad describe nódulos subcutáneos que afectan los miembros inferiores.

La biopsia compatible tanto en piel como en músculo, el curso crónico y recurrente, así como la falta de compromiso de otros órganos, nos llevan al diagnóstico de esta enfermedad poco frecuente.

En cuanto al tratamiento, si bien hay casos descriptos que respondieron adecuadamente al tratamiento con metotrexato, hay que tener en cuenta que no hay evidencia suficiente como para concluir cuál es el mejor.

\section{Conclusión}

Presentamos un caso típico de Panarteritis Nodosa Cutánea, con una localización atípica.

\section{Bibliografía}

1. M.S. Daoud, K.P. Hutton and L.E. Gibson. Cutaneous periarteritis nodosa: a clinic-pathological study of 79 cases. British journal of Dennatology 1997; 136:706713.

2. H Mocan, MC Mocan, H Peru and Y Özoran. Cutaneous polyarteritis nodosa in a child and a review of the literature. Acta Pxdiatr 1998; 87:351-3.

3. Naoko Ishiguro, Makoto Kawashima. Cutaneous polyarteritis nodosa: A report of 16 cases with clinical and histopathological analysis and a review of the published work. Journal of Dermatology 2010; 37:8593.

4. Christina Rogalski, MD, and Michael Sticherling, MD. Panarteritis cutanea benigna - an entity limited to the skin or cutaneous presentation of a systemic necrotizing vasculitis? Report of seven cases and review of the literature. International Journal of Dermatology 2007, 46:817-821.

5. Paulo Ricardo Criado, Gabriela Franco Marques, Thamara Cristiane Alves Batista Morita, Jozélio Freire de Carvalho. Epidemiological, clinical and laboratory profiles of cutaneous polyarteritis nodosa patients: Report of 22 cases and literature review. Autoimmunity Reviews 2016, Volume 15, Issue 6, Pages 558-563.

6. Aaron Joseph Morgan, Robert A Schwartz. Cutaneous polyarteritis nodosa: a comprehensive review. International Journal of Dermatology 2010, 49:750756.

7. María Alejandra Matteoda, Paola Cecilia Stefano, Marcela Bocián, María Marta Katsicas, Josefina Sala, Andrea Bettina Cervini. Cutaneous polyarteritis nodosa. An Bras Dermatol. 2015; 90(3 Suppl 1):S18890.

8. LBreda,SFranchini, VMarzetti,FChiarelli.Intravenous immunoglobulins for cutaneous polyarteritis nodosa resistant to conventional treatment. Scand J Rheumatol $2015 ; 1-2$. 